\title{
Nuclear deformation and neutron excess as competing effects for pygmy dipole strength
}

\author{
R. Massarczyk, ${ }^{1,2}$ R. Schwengner, ${ }^{1}$ F. Dönau, ${ }^{1}$ S. Frauendorf,${ }^{3}$ M. Anders,${ }^{1,2}$ D. Bemmerer, ${ }^{1}$ \\ R. Beyer, ${ }^{1}$ C. Bhatia, ${ }^{4,5},{ }^{4}$ E. Birgersson, ${ }^{1,}$ U M. Butterling, ${ }^{1,}{ }^{6}$ Z. Elekes,${ }^{1}$ A. Ferrari, ${ }^{1}$ \\ M. E. Gooden ${ }^{5,7}$ R. Hannaske ${ }^{1,2}$ A. R. Junghans, ${ }^{1}$ M. Kempe, ${ }^{1,2}$ J. H. Kelley, ${ }^{5,7}$ T. Kögler, ${ }^{1,2}$

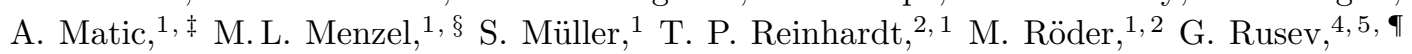 \\ K. D. Schilling, ${ }^{1,2}$ K. Schmidt, ${ }^{1,2}$ G. Schramm, ${ }^{1,2}$ A. P. Tonchev, ${ }^{4,5}$, ** W. Tornow, ${ }^{4,5}$ and A. Wagner ${ }^{1}$ \\ ${ }^{1}$ Helmholtz-Zentrum Dresden-Rossendorf, 01328 Dresden, Germany \\ ${ }^{2}$ Technische Universität Dresden, 01062 Dresden, Germany \\ ${ }^{3}$ University of Notre Dame, Notre Dame, Indiana 46556, USA \\ ${ }^{4}$ Duke University, Durham, North Carolina 27r08, USA \\ ${ }^{5}$ Triangle Universities Nuclear Laboratory, Durham, North Carolina 27708, USA \\ ${ }^{6}$ Martin-Luther Universität Halle-Wittenberg, 06099 Halle, Germany \\ ${ }^{7}$ North Carolina State University, Raleigh, North Carolina 27695, USA
}

(Dated: August 14, 2018)

\begin{abstract}
The electromagnetic dipole strength below the neutron-separation energy has been studied for the xenon isotopes with mass numbers $A=124,128,132$, and 134 in nuclear resonance fluorescence experiments using the $\gamma$ ELBE bremsstrahlung facility at Helmholtz-Zentrum Dresden-Rossendorf and the $\mathrm{HI} \gamma \mathrm{S}$ facility at Triangle Universities Nuclear Laboratory Durham. The systematic study gained new information about the influence of the neutron excess as well as of nuclear deformation on the strength in the region of the pygmy dipole resonance. The results are compared with those obtained for the chain of molybdenum isotopes and with predictions of a random-phase approximation in a deformed basis. It turned out that the effect of nuclear deformation plays a minor role compared with the one caused by neutron excess. A global parametrization of the strength in terms of neutron and proton numbers allowed us to derive a formula capable of predicting the summed $E 1$ strengths in the pygmy region for a wide mass range of nuclides.
\end{abstract}

PACS numbers: 25.20.-x, 25.20.Dc, 21.60.Jz, 24.30.Cz

Photon strength functions (PSF) are important inputs for statistical reaction codes applied in network calculations in nuclear astrophysics and in simulations done for nuclear power production and safety. Knowing and understanding the behavior of the PSF in the energy region around and below the neutron threshold is essential for these applications. For the dominating electric dipole (E1) part of the PSF, the RIPL3 compilation of the IAEA [1] offers an overview on various models, which in essence base on the concept of the damped isovector $E 1$ giant dipole resonance (GDR). It is described by one or two Lorentzian functions with parameters fitted to the characteristic resonance structure observed in $(\gamma, n)$ reactions. For open shell nuclei, which constitute the majority, the nuclear deformation is taken into account. It splits the peak of the GDR [2, 3] and, as a consequence, increases the dipole strength distribution in the region below the neutron-separation energy. Along these lines, a new global description of the PSF was recently presented in Ref. [4], which takes triaxial quadrupole deformation into account and which is called triple Lorentzian model (TLO).

Experimental and theoretical studies [5-9], which have been recently reviewed by Savran, Aumann, and Zilges [10] suggest a richer structure of the PSF below the neutron-separation energy than accounted for by the Lorentzian-type models. In this letter we follow the suggestion in the review [10]: "Today the term Pygmy Dipole Resonance (PDR) is frequently used for the low-lying E1 strength and we will follow this notation in this review without implying with this notation any further interpretation of its structure." The rational behind this terminology is that the interpretation of the PDR depends strongly on the theoretical model invoked and present day experiments cannot distinguish between the models.

One important aspect of studying the PDR concerns its isospin dependence, which is particularly important for simulating the r-process that drives the element synthesis in the cosmic evolution. Experiments including chains of isotopes with changing ratio of neutron-to-proton numbers $N / Z$ address this question. Detailed studies of isotones with the closed neutron shells $N=50$ [1] and $N=82$ [12], respectively, were performed using $\left(\gamma, \gamma^{\prime}\right)$ reactions with bremsstrahlung at the facilities in Dresden and Darmstadt. These studies of spherical nuclides suggested an increase of the pygmy strength with neutron excess. To investigate the effect of nuclear deformation on pygmy strength we studied the even-even Mo isotopes. We found an increase with $N / Z$ [13], which we attributed to the increasing deformation. However, because in the Mo chain the deformation increases along 
with the neutron excess, one cannot disentangle the two mechanisms.

In this Letter, we present the results of a study of the chain of stable even-mass xenon isotopes. In this series of nuclides, the deformation is largest for the lightest isotope and decreases with growing neutron number. We analyze the balance of the effect of nuclear deformation and neutron excess on the strength in the PDR region for the first time and suggest a phenomenological model to describe global trends. The data are compared with calculations in the framework of the deformed Quasiparticle Random Phase (QRPA) approach. Data of the dipole strength in xenon isotopes only exist for the energy region below $4 \mathrm{MeV}$ [14] so far, but neither for the PDR nor for the GDR region, which are also interesting as particular Xe isotopes (e.g. large neutron capture cross section on ${ }^{135} \mathrm{Xe}$ with reasonably short half life) are important as reactor poison for nuclear technology and for the description of nuclear processes in the solar system [15, 16].

The experiments were performed at the bremsstrahlung facility $\gamma \mathrm{ELBE}$ of the HelmholtzZentrum Dresden-Rossendorf [17]. Electrons with an energy $E_{\text {kin }}=12 \mathrm{MeV}$ hit a $7-\mu \mathrm{m}$ thick niobium foil and produce bremsstrahlung with maximum energies exceeding the respective neutron-separation energies. The $\gamma$-ray detection setup consisted of four high-purity germanium detectors surrounded by scintillation detectors acting as escape-suppression shields. The targets consisted of xenon gas of about $2 \mathrm{~g}$, highly-enriched (99.9\%) in the respective isotope, at a pressure of about 80 bar in sphere-shaped steel containers [18] with an inner diameter of $2 \mathrm{~cm}$. They were combined with disks of $0.2 \mathrm{~g}$ of ${ }^{11} \mathrm{~B}$ used for photon flux calibration.

The first important step in the data analysis was the subtraction of the background originating from reactions of photons with the steel container. A comparison of spectra measured for empty and xenon-filled containers is shown in Fig. 1. The spectra of empty and filled containers were normalized by adjusting the peak areas of intense transitions in ${ }^{56} \mathrm{Fe}$. The intensities depend only on the integrated photon flux and are therefore independent of beam fluctuations. The normalization factor derived in this way fits the one deduced from transitions in ${ }^{11} \mathrm{~B}$ with well known cross sections [19, 20].

The analysis included the following steps: (i) Unfolding of detector response and efficiency using GEANT4 [21], (ii) Simulation of the non-nuclear beam-induced background with GEANT4, (iii) Correction for inelastic scattering and estimate of branching ratios using the code $\gamma$ DEX for the simulation of statistical $\gamma$-ray cascades 22, 23. A step-by-step review of the data analysis and uncertainty estimate can be found in Ref. 24]. The total uncertainties are larger compared to previous

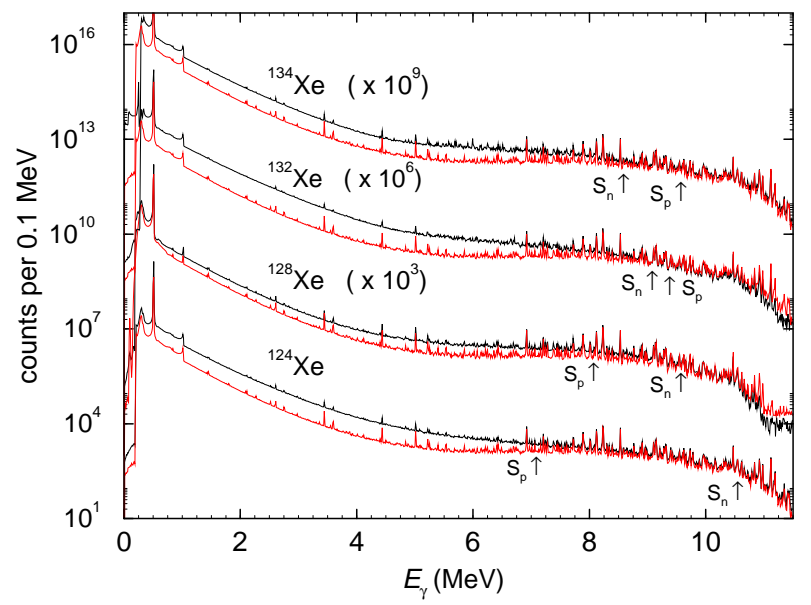

FIG. 1: (Color online) Measured spectra for filled (black) and empty steel containers (red). The spectra of ${ }^{134} \mathrm{Xe},{ }^{132} \mathrm{Xe}$ and ${ }^{128} \mathrm{Xe}$ are scaled up for a better view. The neutron $\left(S_{n}\right)$ and proton $\left(S_{p}\right)$ separation energies are marked with arrows.

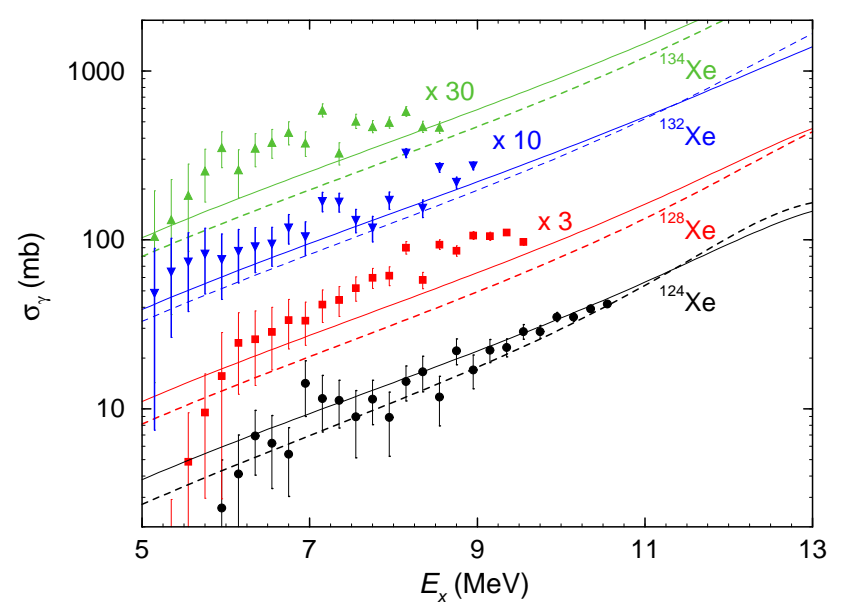

FIG. 2: (Color online) Resulting photo-absorption cross sections for ${ }^{124} \mathrm{Xe}$ (black circles), ${ }^{128} \mathrm{Xe}$ (red squares) ${ }^{132} \mathrm{Xe}$ (blue triangles down), and ${ }^{134} \mathrm{Xe}$ (green triangles up). For comparison predictions of RIPL3 (solid lines) and the TLO (dashed lines) are shown in the corresponding colors. The data for ${ }^{128} \mathrm{Xe},{ }^{132} \mathrm{Xe}$, and ${ }^{134} \mathrm{Xe}$ are multiplied by the factors 3,10 and 30 , respectively.

measurements with solid targets due to the fact that the spectrum of the empty container has to be subtracted.

The photo-absorption cross sections deduced from the experiments at $\gamma$ ELBE are shown in Fig.2, These measured cross sections show an enhancement over the predictions of a two Lorentzian curves, as provided in the RIPL3 database [1] and the triple Lorentzian model [4] in the PDR region.

In addition to these experiments we studied the isotopes ${ }^{128} \mathrm{Xe}$ and ${ }^{134} \mathrm{Xe}$ in experiments at the HighIntensity $\gamma$-ray Source $(\mathrm{HI} \gamma \mathrm{S})$ 25] operated by the Triangle Universities Nuclear Laboratory (TUNL) in Durham, North Carolina. The main aim of these experiments us- 
ing highly polarized quasi-monoenergetic $\gamma$ beams was to identify the contributions of $E 1$ and magnetic dipole M1 strength to the total absorption cross section. The summed $M 1$ strength in the energy region between 6 and $8 \mathrm{MeV}$ was less than $10 \%$ of the $E 1$ strength in the same energy region. The summed magnetic dipole strength between 6 and $8 \mathrm{MeV}$ is $B(M 1)=0.14(3) e^{2} \mathrm{fm}^{2}$ for ${ }^{128} \mathrm{Xe}$ and $B(M 1)=0.07(2) e^{2} \mathrm{fm}^{2}$ for ${ }^{134} \mathrm{Xe}$.

To study a possible effect of nuclear deformation on the dipole strength, we performed calculations in the framework of a quasiparticle-random-phase approximation (QRPA). These calculations take into account quadrupole and triaxial deformation and are therefore suitable for the present task. The QRPA is based on a Wood-Saxon mean field and an isovector dipole-dipole interaction [26]. For the calculation of the low-energy part of the PSF the suppression of the spurious center-of-mass motion was performed as described in Ref. 27].

We transformed the experimental absorption crosssections $\sigma_{\gamma}$ to reduced transition strengths $B(E 1)$ using the relation [2]

$$
4.03 E_{x} B(E 1) \uparrow=\int_{\Delta E} \sigma\left(E_{x}\right) d E \approx \sigma_{\gamma}\left(E_{x}\right) \Delta E
$$

with $B(E 1)$ in $e^{2} \mathrm{fm}^{2}$, the excitation energy $E_{x}$ in $\mathrm{MeV}$, and the absorption cross section $\sigma_{\gamma}$ in $\mathrm{mb}$ in an energy bin $\Delta E=0.2 \mathrm{MeV}$. In the following we will discuss the summed $B(E 1)$ strength in the region of pygmy strength [9], calculated from the interval 6 to $8 \mathrm{MeV}$, (both for theory and experiment).

Fig. 3 shows the summed strengths derived in this way for the Xe isotopes together with the Mo isotopes and the $N=82$ isotones. The general experimental trend is an increase of strength with the ratio $N / Z$. In the case of the Xe isotopes there is almost no change between the heavier isotopes but a remarkable decrease toward the lightest isotope. The increase with $N / Z$ was found in our earlier study of Mo isotopes [13] already.

In addition, we show the $B(E 1)$ strengths for $N=82$ isotones calculated from the data presented in Ref. [12]. The values show a similar increase with $N / Z$, but are considerably smaller than our values for nuclides in the mass-130 region. The reason for this difference is that the values of Ref. 12] only include strength found in resolved peaks. In contrast, the strengths resulting from our analyses include strength in the quasi-continuum of states as well, which amounts to about $60 \%$ of the total strength [24, 28 30]. The smooth connection of the absorption cross section derived in this way with the one known from $(\gamma, n)$ experiments at the neutron threshold proves that it is important to include the strength from the quasi-continuum. Assuming the data of Ref. [12] underestimate the total strength by the same factor, the general trend of increase is also apparent.

Fig. 3] compares the experimental strength with the calculated QRPA and TLO values. The details of the QRPA

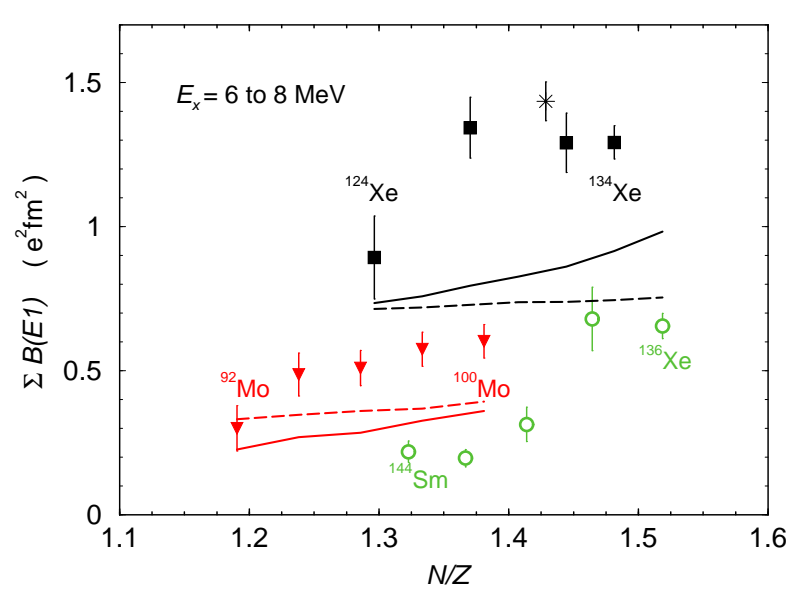

FIG. 3: (Color online) Summed $B(E 1) \uparrow$ values versus neutron-to-proton number. The $\gamma \mathrm{ELBE}$ data for the four xenon isotopes (black squares) and for ${ }^{136} \mathrm{Ba}$ (black asterisk) are shown in comparison with the $\gamma \mathrm{ELBE}$ data for the molybdenum isotopes [13] (red triangles) and with results for ${ }^{136} \mathrm{Xe}$ and further $N=82$ isotones from other experiments [12] (open green circles). Further, the results of the QRPA calculations for all stable even-mass xenon (black solid line) and molybdenum isotopes (red solid line) as well as the TLO predictions (dashed lines) are shown.

calculations are described in Ref. 26, 31], where the deformation parameters $\beta_{2}$, which result from the micromacro mean field calculations, are quoted. These values are consistent with the experimental ones given in Ref. 32] and used also as input deformation of the TLO model. The QRPA calculations reproduce the gross dependence on $N / Z$. However, the scale of pygmy strength is underestimated, which seems to be a general problem with our version QPRA that already came up in our earlier studies [24, 28]. At this point, we can only speculate about the reason. One possibility is a certain degree of collectivity in the pygmy region (the celebrated vibration of the neutron skin against the core with equal neutron and proton numbers [9]), which is not accounted for by our simple dipole-dipole interaction. Another possibility are complex excitations beyond the QRPA, as for example fragmented two-phonon quadrupole-octupole states. The TLO values, which depend very weakly on $N / Z$, are also too low. The similar scale of QRPA and TLO may not be by accident. Both models incorporate the isovector dipole vibrations, obey the TRK E1 sum rule [3335], and introduce a spreading width that ensures that the width of the GDR peak is reproduced (see [4, 31]).

In order to expose the effects of the nuclear quadrupole deformation the pygmy strengths are replotted in Fig.4 versus the deformation parameter $\beta_{2}$. The Mo chain shows a smooth increase of pygmy strength with deformation. In this case the growing deformation combines constructively with the increasing neutron excess. The QRPA well accounts for the increase, whereas the TLO 


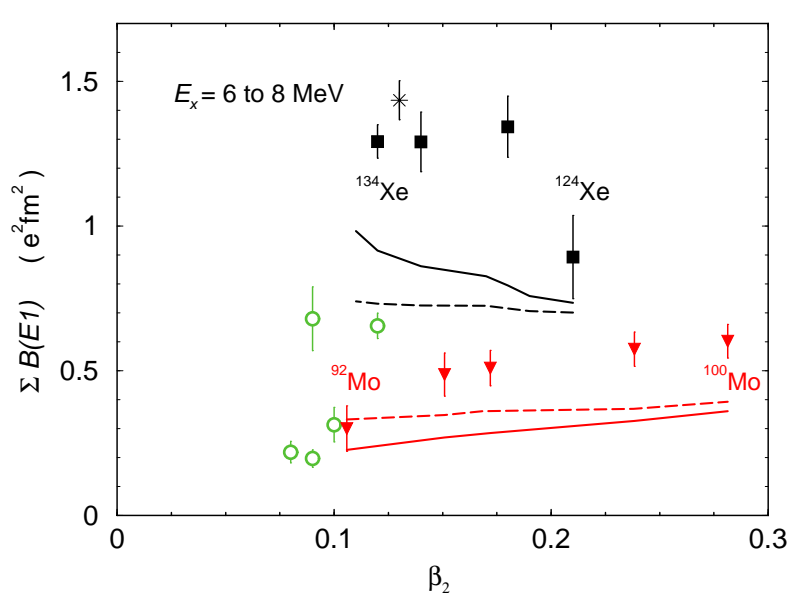

FIG. 4: (Color online) Summed $B(E 1) \uparrow$ values for different isotopes in the $N=50$ and $N=82$ region over the nuclear quadrupole deformation parameter $\beta_{2}$. The definition of the symbols corresponds to the one in Fig. 3.

underestimates it. In the case of the Xe isotopes the combination is destructive. The values reach from $\beta_{2} \approx 0.21$ for ${ }^{124}$ Xe with the smallest $N / Z$ to $\beta_{2} \approx 0.1$ for the heavy isotopes. The Xe isotopes show a decrease of the pygmy strength with deformation: almost constant values for the nuclides with little deformation and a smaller value for the deformed ${ }^{124} \mathrm{Xe}$, which is the most remarkable finding of this Letter. The QRPA calculations qualitatively reproduce the decrease with increasing deformation. The TLO values stay rather constant with a tiny decrease toward larger deformation. The latter is the result of the competition between an increase caused by deformation and a decrease caused by the $N Z / A$ dependence of the TRK on the neutron excess. The QRPA strengths are much more sensitive to deformation than the TLO ones, which turns out to be important for reproducing the experimental trends. This indicates that the shell structure, which is taken into account in QRPA but not in TLO, strongly influences the deformation dependence of the $E 1$ strength.

As a result of this analysis, we conclude that the deformation has only a minor effect on the summed E1 strength in the PDR region and that it is sensitive to the local shell structure. This suggests that the neutron excess is the key element for the global $N-Z$ dependence of the strength below the neutron separation threshold. So far, it has been studied only for spherical nuclei, in experiments as well as in RQTBA [24], QRPA and QPM[11] calculations. To quantify the global trends, we parametrize the integrated $E 1$ strength in terms of $N$ and $Z$ neglecting the dependence on the deformation. Fig. 3. suggests an approximately linear dependence on the ratio $N / Z$

$$
\sum B(E 1) \propto\left(\frac{N}{Z}-1\right)
$$

The ratios of the pygmy strength between the different isotopes are well described by the $N-Z$ dependence of the TRK sum rule, $N Z / A$. Combining these observation results in the expression

$$
\sum_{6-8 \mathrm{MeV}} B(E 1) \approx r \frac{N Z}{A}\left(\frac{N}{Z}-1\right)
$$

for the summed strength in $e^{2} \mathrm{fm}^{2}$ in the interval from 6 to $8 \mathrm{MeV}$. To check the quality of the approximation and fix the parameter $r$, we calculated the ratios $r_{E 1}$ of the experimental and parametrized pygmy strengths

$$
r_{E 1}=\sum B(E 1)\left[\left(\frac{N}{Z}-1\right) \frac{N Z}{A}\right]^{-1} .
$$

These ratios stay rather constant for the considered Mo, $\mathrm{Xe}$ and $\mathrm{Ba}$ isotopes around an average value of $r_{E 1}=$ $0.080(5)$, which determines the free parameter in Eq. (3) to $r \approx 0.08$ in Eq. (3).

We tested Eq. (3) for nuclides with masses up to 250 using experimental data available in the EXFOR database [36]. The comparison of the predictions of Eq. (3) with experimental $B(E 1)$ values deduced from the cross sections given in EXFOR according to Eq. (11) is shown in Fig.5. We find a generally good agreement in the whole considered mass region including light nuclides as well as very heavy nuclides such as uranium and thorium isotopes which are well deformed. The general agreement supports our finding that deformation has a minor influence on the low-lying dipole strength on the global scale.

As a caveat, we notice that the summed $E 1$ strength of double magic nuclei, such as ${ }^{208} \mathrm{~Pb}$, cannot be described with Eq. (3), probably because of their exceptional small fragmentation of the low-lying strength (see also Ref. [37]). Further, the formula fails for nuclides with $N=Z$. For practical purposes, this is of minor importance because the valley of stability does not follow the $N=Z$ line and applications in nuclear technology as well as in nuclear astrophysics concern mainly neutronrich nuclei.

Summarizing, we analyzed the dipole strength in the $\mathrm{PDR}$ region in the chain of xenon isotopes. In contrast to our earlier study of molybdenum isotopes we could disentangle the effects of neutron excess and nuclear deformation on the dipole strength and study their competition for the first time. We found the neutron excess to have the dominating effect on the strength below the neutron separation energy. We constructed a simple parametrization of the summed PDR strength that describes its global $N$ and $Z$ dependence in a wide mass range of nuclides.

We thank A. Hartmann for technical support and the crews of the ELBE accelerator and of the HI $\gamma \mathrm{S}$ facility for their collaboration during the experiments. This 


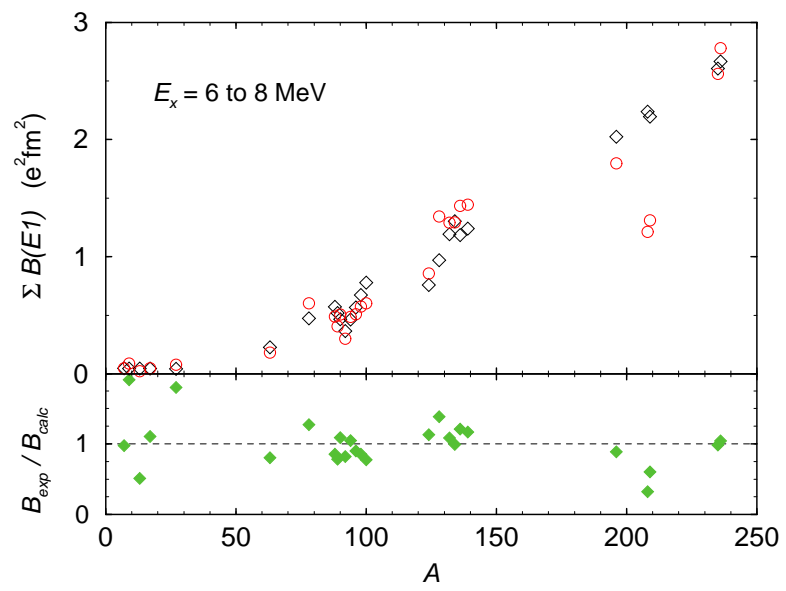

FIG. 5: (Color online) Comparison of experimental data (red circles) and the prediction by Eq. (3) (black diamonds). The bottom subfigure shows the divergence of the two.

work was supported by the German Research Foundation (DFG), project no. SCHW883/1-1 and partly by the EURATOM FP7 Project ERINDA (FP7-269499). Partial support was also granted by the U.S. Department of Energy, Office of Nuclear Physics, under Grants No. DE-FG02-95ER4093, No. DE-FG02-97ER41033 and No. DE-FG02-97ER41042.

* Present address: McMaster University, Hamilton, Ontario, Canada.

† Present address: Areva NP GmbH, 91052 Erlangen, Germany.

¥ Present address: IBA Particle Therapy, 45157 Essen, Germany.

$\S$ Present address: Max Planck Institut für Extraterrestrische Physik, 85741 Garching, Germany.

ฯ Present address: Los Alamos National Laboratory, Los Alamos, New Mexico 87545, USA.
** Present address: Lawrence Livermore National Laboratory, Livermore, California 94550, USA.

[1] R. Capote et al., Nuclear Data Sheets 110, 3107 (2009).

[2] A. Bohr and B. R. Mottelson, Nuclear structure, vol. II, (W. A. Benjamin, Inc., Reading, Massachusetts, 1975).

[3] J. M. Eisenberg and W. Greiner, Nuclear theory, vol. I, (North-Holland, Amsterdam, 1975), chs. 10 and 11.

[4] A.R. Junghans et al., Phys. Lett. B 670, 200 (2008).

[5] A.M. Lane, Ann. Phys. (N.Y.) 63, 171 (1971).

[6] S. Volz et al., Nucl. Phys. A779, 1 (2006).

[7] A. Zilges et al., Phys. Lett. B 542, 43 (2002).

[8] A. Tonchev et al., Phys. Rev. Lett. 104, 072501 (2010).

[9] N. Tsoneva, H. Lenske, and Ch. Stoyanov, Phys. Lett. B 586, 213 (2004).

[10] D. Savran et al., Prog. Part. Nucl. Phys. 70, 210 (2013).

[11] R. Schwengner et al., Phys. Rev. C 87, 024306 (2013).

[12] D. Savran et al., Phys. Rev. Lett. 100, 232501 (2008).

[13] G. Rusev et al., Phys. Rev. C 79, 061302 (2009).

[14] H. von Garrel et al., Phys. Rev. C 73, 054315 (2006).

[15] J. Kunz et al., Science 280, 877 (1998).

[16] S. Mukhopadhyay, Nature 486, 101 (2012).

[17] R. Schwengner et al., Nucl. Instr. Meth. A 555, 211 (2005).

[18] G. Rupp et al., Nucl. Instr. Meth. A 608, 152 (2009).

[19] F. Ajzenberg-Selove, Nucl. Phys. A 506, 1 (1990).

[20] G. Rusev et al., Phys. Rev. C 79, 047601 (2009).

[21] S. Agostinelli et al., Nucl. Instr. Meth. A 506, 250 (2003).

[22] G. Schramm et al., Phys. Rev. C 85, 014311 (2012).

[23] R. Massarczyk et al., Phys. Rev. C 87, 044306 (2013).

[24] R. Massarczyk et al., Phys. Rev. C 86, 014319 (2012).

[25] H.R. Weller et al., Prog. Part. Nucl. Phys. 62, 257 (2009).

[26] I. Bentley et al., Phys. Rev. C 83, 014317 (2011).

[27] F. Dönau, Phys. Rev. Lett. 94, 092503 (2005).

[28] A. Makinaga et al., Phys. Rev. C 82, 024314 (2010).

[29] G. Rusev et al., Phys. Rev. C 77, 064321 (2008).

[30] R. Schwengner et al., Phys. Rev. C 78, 064314 (2008).

[31] S. Q. Zhang et al., Phys. Rev. C 80, 021307 (2009).

[32] S. Raman et al., Atomic Data Nucl. Data Tables 78, 1 (2001).

[33] W. Thomas, Naturwissenschaften 13, 627 (1925).

[34] F. Reiche and W. Thomas, Z. Phys. 34, 510 (1925).

[35] W. Kuhn, Z. Phys. 33, 408 (1925).

[36] http://www.nndc.bnl.gov/exfor/exfor00.htm.

[37] R. Schwengner et al., Phys. Rev. C 81, 054315 (2010). 